\title{
Contribuições do enfermeiro para o cuidado de crianças com estomia intestinal no
}

\section{âmbito escolar}

Nurses' contribuitions to care of children with intestine stomy at school

\author{
Contribuciones de las enfermeras al cuidado de niños com estomia intestinal en la escuela
}

Larissa Christiny Amorim dos Santos ORCID: https://orcid.org/0000-0002-9705-5811 Universidade Iguaçu, Brasil

E-mail: amorimlari224@gmail.com

Wanderson Alves Ribeiro

ORCID: https://orcid.org/0000-0001-8655-3789 Universidade Iguaçu, Brasil

E-mail: nursing_war@hotmail.com

Bruna Porath Azevedo Fassarella ORCID: https://orcid.org/0000-0002-1400-4147 Universidade Iguaçu, Brasil

E-mail: brunaporath@gmail.com

Keila do Carmo Neves

ORCID: https://orcid.org/0000-0001-6164-1336 Universidade Iguaçu, Brasil

E-mail: keila_arcanjo@hotmail.com

Ana Lúcia Naves Alves

ORCID: https://orcid.org/0000-0003-0791-5775 Universidade Iguaçu, Brasil

E-mail: ananaves.alna@gmail.com

Kemely de Castro

ORCID: https://orcid.org/0000-0003-0462-3312 Universidade Iguaçu, Brasil

E-mail: kemely.8castro@gmail.com

Pedro Oscar Lopes Salvati

ORCID: https://orcid.org/0000-0001-5765-5030 Universidade Iguaçu, Brasil

E-mail: lopespedrooscar@gmail.com

Douglas Henrique Serejo da Silva ORCID: https://orcid.org/0000-0003-1914-818X Universidade Iguaçu, Brasil

E-mail: dhsdouglas00@gmail.com

\section{Resumo}

O presente estudo se trata de uma pesquisa reflexiva sobre as contribuições do enfermeiro para o cuidado de crianças com estomias no âmbito escolar. Realizadas nas bases de dados da Biblioteca Virtual de Saúde (BVS), na seguinte base de informação: Literatura Internacional em Ciência da Saúde (MEDLINE), Scientific Eletronic Library Online (SCIELO). As Crianças com estomias intestinais, embora tenham características que as unem em uma condição especial, estão em constante desenvolvimento e suas familias necessitam de adequação a esse novo estilo de vida. Vale ressaltar que o enfrentamento dessa necessidade especial, acarretará diversas modificações no dia a dia. Sendo de fundamental importância a implementação de um serviço oferecido por um profissional competente e capacitado para orientar as crianças estomizadas e seus familiares sobre os cuidados, implementando um serviço holístico e diferenciado. Diante do exposto, pode-se refletir sobre a valorização desses enfermeiros e os especialistas em Estomaterapia, sendo eles capacitados para tal atividade. Vale refletir também, a possibilidade de empregar essas orientações aos acadêmicos, pois quanto mais cedo for orientado, melhor será o seu êxito frente a essa situação.

Palavras-chave: Estomia; Papel do enfermeiro; Criança.

\begin{abstract}
This study is a reflective research on the contributions of nurses to the care of children with ostomy in the school environment. Carried out in the Virtual Health Library (VHL) databases, in the following information base: International Health Science Literature (MEDLINE), Scientific Electronic Library Online (SCIELO). Children with intestinal ostomies, although they have characteristics that unite them in a special condition, are in constant development and their families need to adapt to this new lifestyle. It is noteworthy that facing this special need will lead to several
\end{abstract}


changes in daily life. It is of fundamental importance to implement a service offered by a competent and qualified professional to guide ostomized children and their families about care, implementing a holistic and differentiated service. Given the above, one can reflect on the appreciation of these nurses and specialists in Stomatherapy, who are trained for such activity. It is also worth reflecting on the possibility of using these guidelines to students, because the sooner they are guided, the better their success will be in this situation.

Keywords: Ostomy; Nurse's role; Kid.

\section{Resumen}

Este estudio es una investigación reflexiva sobre los aportes de las enfermeras al cuidado de los niños con ostomía en el ámbito escolar. Realizado en las bases de datos de la Biblioteca Virtual en Salud (BVS), en la siguiente base de información: Literatura Internacional en Ciencias de la Salud (MEDLINE), Biblioteca Electrónica Científica en Línea (SCIELO). Los niños con ostomía intestinal, aunque tienen características que los unen en una condición especial, están en constante desarrollo y sus familias necesitan adaptarse a este nuevo estilo de vida. Es de destacar que hacer frente a esta necesidad especial conllevará varios cambios en la vida diaria. Es de fundamental importancia implementar un servicio ofrecido por un profesional competente y calificado para orientar a los niños ostomizados y sus familias en el cuidado, implementando un servicio holístico y diferenciado. Dado lo anterior, se puede reflexionar sobre la apreciación de estos enfermeros y especialistas en Estomatoterapia, quienes están capacitados para tal actividad. También vale la pena reflexionar sobre la posibilidad de aplicar estas pautas a los alumnos, pues cuanto antes se orienten mejor será su éxito en esta situación.

Palabras clave: Ostomía; Papel de la enfermera; Niño.

\section{Introdução}

De origem grega, a palavra "estomia" ou "estoma" significa boca ou abertura de quaisquer vísceras oca no corpo criada cirurgicamente. E sua denominação dependerá do órgão que será exteriorizado, e com objetivo de alterar o trânsito intestinal para eliminação de efluentes (fezes) (Mafra, 2020).

Se consolidando ao longo do tempo, a portaria n ${ }^{\circ} .400$ de 2009, estabelece as Diretrizes Nacionais para a criação de Serviço de Atenção à Saúde das Pessoas Ostomizadas (SASPO) no âmbito do Sistema Único de Saúde (SUS) (Paula, 2020).

A descoberta de malformações congênitas são um dos motivos mais comuns para a criação de estomias em crianças. Sendo vista como algo amedrontador e tendo um efeito devastador na vida desses pacientes, trazendo medos, angústias, incertezas e inseguranças principalmente para seus responsáveis (Simon, 2020).

Dentre as malformações frequentes, se destacam as anomalias anorretais como imperfuração anal; megacólon e a atresia intestinal. Acarretando em uma enterostomias e consequentemente a necessidade do uso de bolsas coletoras (Barros et al., 2020).

Conforme o grau e etiologia de sua gravidade, o cirurgião indicará a prática de estomias, podendo ser provisório ou definitivo. Com isso, essas estomias causam alguns prejuízos, acarretando baixa autoestima, mudanças na fisiologia gastrintestinal, mudanças sociais e afetivas de seus pacientes (Diniz et al., 2020).

Em estudos referentes sobre a principal causa de indicação cirúrgica para realização de estomia, se destaca doença de Hirschsprung ou megacólon. Sendo ela caracterizada por incidência de cerca 1:5000 nascidos vivos, é definida por uma má formação do intestino grosso e caracterizada pela ausência de células ganglionares na extremidade distal do intestino (Almeida et al., 2021).

Seus familiares enfrentam diferentes dificuldades, não só pela necessidade de se adequar ao novo estilo de vida, que antes não faziam parte do seu cotidiano para bem-estar dessa criança, mas também pela dificuldade financeira e psicologia (Mendonça, 2018).

Após a cirurgia, a criança não conseguirá mais controlar a eliminação de efluentes voluntariamente e, devido a isso, precisará utilizar bolsas coletoras especiais, as quais são adaptadas no abdome. Necessitando de cuidados indispensáveis, como higiene adequada e manutenção da integridade da pele (periestoma), prevenindo infecções, traumas, necrose, entre outras (Melo et al., 2020). 
Crianças com estomias intestinais, embora tenham características que as unem em uma condição especial, estão em constante desenvolvimento e apresentam singularidade em cada fase com desigualdade social, biológicas, e emocionais exigindo uma atenção e cuidado diferenciado.

O enfrentamento dessa necessidade especial, acarretará diversas modificações no dia a dia. Sendo de fundamental importância a implementação de um serviço oferecido por um profissional competente, capacitado para orientar as crianças estomizadas, familiares e cuidadores sobre os cuidados com a saúde. Na equipe multiprofissional o enfermeiro desempenha papel fundamental no cuidado deste paciente, pois ele está presente em todos os serviços de saúde desde unidades básicas de saúde até os de maior complexidade.

\section{Metodologia}

Trata-se de pesquisa descritiva com abordagem reflexiva. Realizadas nas bases de dados da Biblioteca Virtual de Saúde (BVS), na seguinte base de informação: Literatura Internacional em Ciência da Saúde (MEDLINE), Scientific Eletronic Library Online (SCIELO), dentre outros.

Optando-se pelos seguintes descritores: Estomia; Papel do Enfermeiro; Criança. Utilizou-se a análise temática para o aprofundamento das discussões e como fonte apenas artigos que tiveram estreita relação com objetivo deste estudo. Para tanto foram incluídos na busca experiências de crianças estomizadas com a faixa etária entre 0 a 6 anos em fase pré-escolar e relatos de familiares e professores. Como critérios de exclusão, estudos que não abordassem a temática estabelecida e com recorte temporal inferior a 2017.

\section{Resultados e Discussão}

Tratando-se de crianças com estomias, existe a necessidade de orientação dos profissionais de saúde aos cuidadores dessas crianças com estomas. O presente estudo caracteriza-se por seus dados serem de relevância, cuja temática retrata a dificuldade vivenciada por crianças estomizadas. Visando identificar as estratégias de enfermagem para promoção do cuidado familiar da criança com estomia intestinal e ressaltar a importância desse profissional no atendimento. A análise temática realizada emergiu quatro categorias: (i) As dificuldades dos familiares diante da nova situação; (ii) Inserção escolar de criança com estomia; (iii) Contribuições do enfermeiro no cuidado de crianças estomizadas; (iv) Importância da enfermagem na orientação e acompanhamento no cuidado da criança estomizada.

\subsection{As dificuldades dos familiares diante da nova situação}

No que se refere a vivência do familiar com a criança com estomia, se dá aos diversos obstáculos como o julgamento da população, a aceitação do familiar perante a situação atual do filho e as dificuldades no cuidado que será prestado diariamente (Couto et al., 2021).

Em uma pesquisa feita com familiares de crianças com estomia, revela que um dos desafios enfrentados, é a bolsa de colostomia, sendo um longo processo adaptativo. O processo de cuidado de uma criança com estomia é de contínua mudança, podendo afetar suas relações, sendo a figura materna, na maioria das vezes, a principal cuidadora (Koeppe \& Cirino, 2020).

Diante da nova realidade, o familiar precisará se adequar, se deparando com exigências e sobrecarga que pode acarretar problemas emocionais e estressantes. Onde muitas vezes carecem de acompanhamento psicológico para lidar com a situação (Almeida et al., 2020). 
A rede de apoio se torna de grande valia não só para o adequado desenvolvimento da criança, mas para garantia da unidade familiar frente as dificuldades enfrentadas. Compreendendo que essa rede seja fundamental, pois muitas vezes quem está estomizado se encontra com o emocional fragilizado (da Silva et al., 2020).

Estabelecido pela Portaria n ${ }^{\circ}$ 400, as diretrizes Nacionais para a Atenção à Saúde das Pessoas Estomizadas, fornece o atendimento, prescrição e adequação de equipamentos coletores e adjuvantes de proteção e segurança, garantindo o acesso á saúde (Faria \& Kamada, 2020).

No âmbito familiar, prevalecem o desconhecimento e o despreparo para lidar com a criança com estomia devido a falta de material adequado, falta de orientação a orientação inadequada. Por isso é fundamental que os profissionais oriente-os de forma correta, de modo que os pais, e familiares estejam aptos na realização desse cuidado (da Silva \& da Fonseca, 2020).

Desse modo, nota-se que apesar das dificuldades vivenciadas, existem leis que respaldam esse paciente e seus entes. Ressalta-se ainda que as redes de apoio são fundamentais, além do convívio com outras crianças estomizadas, tornando uma opção para adquirir conhecimentos através de trocas de experiências, e a medida que o elo de confiança se fortalece, fazendo com que haja motivação para o enfrentamento dos desafios.

\subsection{Inserção escolar de criança com estomia}

Durante muitos anos, a educação se destinava apenas para pessoas dentro de um padrão de normalidade. Porém, com o intuito de tornar as escolas um lugar de inclusão e menos desigual, muitas mudanças ocorreram.

A LEI Nº 13.146, DE 6 DE JULHO DE 2015, reforça que a educação inclusiva é para todos os estudantes. Garantindo direitos e promovendo a aprendizagem, estimulando a autonomia e a independência das pessoas com deficiência em todas as fases da vida, tendo como foco nesse contexto, as crianças com estomia (Lucas, 2021).

As escolas são importantes na fase de pré-alfabetização, possibilitando aprendizagens diante das limitações impostas pela situação que ela se encontra, alcançando o mais completo progresso educacional, além da inclusão social (Fidalgo, 2021).

Em pesquisas feitas sobre os sentimentos vivenciados pelos professores no âmbito escolar, se destacam o medo, insegurança e preocupação. Onde muitos revelaram nunca ter tido nenhum tipo de orientação, curso ou instrução da secretaria de educação ou saúde para tais cuidados (Melo et al., 2017).

Nesse sentido, torna-se fundamental que os professores contem com uma rede de apoio, e com educadores de saúde preferencialmente, para auxiliá-lo no desenvolvimento de seu trabalho e consequentemente, tornar o âmbito escolar uma rede de inclusão (Camargos et al., 2021).

Diante do exposto, faz-se relevante conhecer as implicações do atendimento à criança com estomia no contexto escolar, além do familiar, anteriormente explicitado. Ressaltando assim, a importância da educação em saúde para essa classe de profissionais, oferecendo informações sobre o cuidado, limpeza, além da oferta do apoio que se torna fundamental nessa faixa etária.

\subsection{Contribuições do enfermeiro no cuidado de crianças ostomizadas}

A especialidade exclusiva do enfermeiro voltada para o cuidado a pessoas ostomizadas, com lesões de pele e incontinência anal ou urinária é a estomaterapia. As funções do estomaterapeuta vem sendo debatidas e comprovadas, sendo ele, o profissional capacitado com conhecimentos e treinamento específico (Paixão, 2020).

Essa especialidade surgiu nos Estados Unidos, no final da década de 1950. No Brasil a especialidade teve início oficial com a criação do I Curso de especialização (lato sensu) em Enfermagem em estomaterapia, na Escola de Enfermagem da Universidade de São Paulo (USP), em 1990 (Vieira, 2018). 
Outro fato relevante é a conquista da Portaria $\mathrm{n}^{\circ} 620$, de 12 de novembro de 2010, onde integra o enfermeiro estomaterapeuta na tabela de classificação do Sistema de Cadastro Nacional de Estabelecimento de Saúde (SCNES) (Costa et al., 2020).

O estomaterapeuta pode atuar nos ambulatórios, serviços públicos e privados, clínicas, consultórios especializados em estomaterapia e assistência domiciliar, ganhando destaque na saúde. Sendo capaz de acompanhar esse indivíduo desde os primeiros cuidados, orientando o paciente visando torná-lo independente para continuidade do cuidado em sua residência (Wojastyk et al., 2020).

Todavia, o cuidado com as feridas, incontinências e estomas não são atividades exclusivas apenas do estomaterapeuta. Por isso, cabe ao enfermeiro generalista o aprofundamento nessa temática. Sendo ele capaz de identificar fragilidades, medos, receios e dúvidas, gerando confiança entre o profissional, o familiar e a criança ostomizada (de Oliveira et al., 2020).

Diante do exposto, pode-se refletir sobre valorização desses enfermeiros e os especialistas em estomaterapia, sendo eles capacitados para tal atividade. Vale refletir também, a possibilidade de empregar essas orientações aos acadêmicos, pois quanto mais cedo for orientado, melhor será o seu êxito frente a essa situação (da Silva \& Leite, 2020).

\subsection{Importância da enfermagem na orientação e acompanhamento no cuidado da criança ostomizada}

Quando se trata de uma criança estomizada, os familiares necessitam de orientações especializadas, através de estratégias educativas objetivando uma aproximação e confiança para o enfrentamento dessa nova situação (da Silva \& Leite, 2020).

O enfermeiro é o profissional de saúde capacitado para prestar um atendimento de qualidade para a criança e o familiar, atendendo as necessidades individuais e promovendo as orientações para uma participação (Gonzaga et al., 2020).

O serviço prestado deverá ser um suporte assistencial, carecendo de atualizações e formulações de estratégias para o aprimoramento de suas técnicas, como também na aproximação familiar (Barros et al., 2020).

As orientações ofertadas por enfermeiros generalistas ou estomaterapeutas deverão ser pautadas de acordo com as características da estomia, se atentando ao equipamento coletor que mais se adapta de acordo com a idade da criança e o uso de adjuvantes quando forem necessários (Paixão, 2020).

É indispensável que os enfermeiros elaborem um plano terapêutico e holístico, com avaliação frequente da região periestomal e da estomia, para que a assistência seja prestada de maneira integral (Silva, 2020).

Deste modo, ressalta-se a participação fundamental do enfermeiro na prestação do cuidado ao estomizado, orientação e suporte. A consolidação do cuidado integral aos estomizados requer capacitação especializada com a finalidade de capacitar o manejo evitando possíveis complicações.

\section{Conclusão}

Através do estudo supracitado, fica evidente a deficiência de materiais voltados a essa temática. Sendo de importância a formulação de estratégias para orientação da população, visando o aprimoramento do conhecimento que influência diretamente em uma prática adequada no atendimento.

Conclui-se que os familiares enfrentam diversas dificuldades tanto na aceitação como na mudança para novos hábitos visando se adequar a nova realidade, além da falta de conhecimento dos professores sobre essa causa, reforçando o sentimento de medo, receio e preocupação que são vivenciadas diariamente por todos aqueles ao redor daquela criança. Tornando o enfermeiro o profissional capacitado para prestar atendimento especializado e individualizado para esses pacientes, orientando e estimulando-os para uma melhor qualidade de vida. 
Além de deixar evidente a possibilidade de empregar as orientações aos acadêmicos, pois quanto mais cedo for orientado, melhor será o seu êxito. Tornando os cuidados para os ostomizados mais acessível para todos.

Através desse estudo, espera-se contribuir para melhoria das ações de enfermagem frente aos cuidados em crianças com estomias intestinais além de estimular a elaboração de outros estudos relacionados a esta temática.

\section{Referências}

Almeida, A. R., Alves, V. H., da Costa Vidal, D. L., Pereira, A. V. \& Vieira, B. D. G. (2020). O cuidado de saúde de crianças estomizadas: uma revisão integrativa da literatura. Research, Society and Development. 9 (10), e849108271-e849108271.

Almeida, A. R., da Costa Vidal, D. L., Alves, V. H., \& Pereira, A. V. (2021). O acesso de crianças com estomia aos serviços especializados de saúde no município do Rio de Janeiro. Scientific Eletronic Library Online. https://doi.org/10.1590/SciELOPreprints.2880

Barros, M. C. A. D., Queiroz, A. A. R. P., Sianfaran, V. G., \& Aguiar, A. A. (2020). Perfil de pacientes pediátricos estomizados atendidos em um hospital público de Pernambuco. Faculdade pernanbucana de Saúde, 34

Camargos, G. R. S., Ferreira, J. M., do Carmo, R. A. B., \& Alves, R. M. (2021). As Pessoas com deficiência e o papel da inclusão nas escolas. Revista Projetos Extensionistas, 1(1), 132-145

Cirino, H. P., Kestenberg, C. C. F., Caldas, C. P., do Nascimento Santos, C. \& Ribeiro, W. A. (2020). Repercussões emocionais e processos adaptativos vividos por pessoas estomizadas. Saúde Coletiva (Barueri), 10(57), 3573-3596.

Costa, C. C. P. D., Soares, S. S. S., Vieira, M. L. C., Oliveira, M. D., Pedro, R. S., Chaves, U. S. B. \& Souza, N. V. D. D. O. (2020). Estomaterapeutas no mundo do trabalho: facilidades e dificuldades para o exercício profissional. Escola Anna Nery, 25.

Couto, J. A., de Sá, T. D. S., da Silva, K. D. S. \& Nunes, M. R. (2021). Orientações de enfermagem a pacientes ostomizados: Revisão integrativa. Research, Society and Development, 10 (9), e31310918086-e31310918086.

da Silva, E. A. \& Leite, V. C. V. (2020). Empreendedorismo em enfermagem e protagonismo do enfermeiro estomaterapeuta: revisão integrativa. Revista Remecs-Revista Multidisciplinar de Estudos Científicos em Saúde, 32.

da Silva, A. L. R., \& da Fonseca, M. I. (2020). Atendimento à pessoa ostomizada. Revista Fontes Documentais, 3, $528-536$.

da Silva, T. P., Silva, Í. R., da Silva, L. J. Ferreira, M. J. C., Moreira, M. C., \& Pinto, C. B. (2020). Criança com estoma nos estudos de Pós-Graduação Stricto Sensu em Enfermagem: destacando aspectos epistemológicos. Revista Enfermagem UERJ, $28,48514$.

de Mendonça, C. R. L. F. (2018). Sobre ocupar-se de cuidar do filho no hospital: o que dizem as mães de crianças cardiopatas? Revista de Terapia Ocupacional da Universidade de São Paulo, 29(3), 263-269

de Oliveira Carvalho, S., Moura, M. C. S., Mesquita, M. K. R., da Silva, G. A. D. A, Vasconcelos, C. D. A., \& da Silva, G. R. F. (2021). Ações extensionistas em estomaterapia: Relato de experiência durante a pandemia. Research, Society and Development, 10 (9), e54010918223-e54010918223.

Diniz, I. V., Costa, I. K. F., Nascimento, J. A., Silva, I. P. D., Mendonça, A. E. O. D. \& Soares, M. J. G. O. (2021). Fatores associados à qualidade de vida de pessoas com estomas intestinais. Revista da Escola de Enfermagem da USP, 55.

Faria, T. F., \& Kamada, I. (2020). Complicações de estomias e perfil clínico de crianças atendidas em um hospital de referência. Estima-Brazilian Journal of Enterostomal Therapy, 18 .

Fidalgo, S. S. (2021). A linguagem da exclusão e inclusão social na escola. Editora Unifesp.

Gonzaga, A. C., Albergaria, A. K. A., Araújo, K. O. P., Borges, E. L. \& Junior, J. F. P. (2020). Perfil de crianças e adultos com estomia intestinal do centro de referência da Bahia - Brasil. Estima (online), e0520-e0520.

Koeppe, G. B. O., Ferreira, A. D., Soares, J. S., Cerqueira, L. D. C. N., da Paz Torres, V. C., \& Oliveira, P. P. (2020). Perfil clínico e demográfico de crianças e adolescentes portadores de estomia atendidos em serviço de referência-Clinical and demographic profile of children and adolescents with ostomy treated at a reference service. Revista Eletrônica de Ciência, Tecnologia e Inovação em Saúde, 1, 55-66.

Lucas, L. M. (2021). A Lei Brasileira de Inclusão da Pessoa com Deficiência (Lei n. 13.146/2015) e as inovações na teoria das incapacidades: um estudo acerca do regime de validade do negócio jurídico celebrado por pessoa com deficiência intelectual. https://repositorio.ufsc.br/handle/123456789/228684

Mafra, I. F. (2020). Estudo da efetividade da demarcação de estoma intestinal por estomaterapeuta em pacientes com doença oncológica. 119 f. Dissertação (Mestrado em enfermagem) - Universidade Federal do Amazonas, Manaus.

Melo, M. C., Kamada, I., Dutra, L. M. A., Simões, J. F. F. L. \& de Melo, E. M. O. P. (2017). Vivência do professor no cotidiano da criança com estomia: abordagem da Fenomenologia Social. Revista Eletrônica de Enfermagem, 19.

Melo, M. C., Vilas-Boas, B. N. F., Martins, B. L., Vasconcellos, A. W. A. \& Kamada, I. (2020). Práticas no cuidado à criança estomizada: narrativas de familiares. Revista Brasileira de Enfermagem, 73.

Paixão, A. R. D. (2020). Atuação do enfermeiro estomaterapeuta na atenção domiciliar: A importância do profissional desde o atendimento ao paciente até a gestão junto as operadoras de saúde. Revista Estima. 
Research, Society and Development, v. 10, n. 15, e423101523077, 2021

(CC BY 4.0) | ISSN 2525-3409 | DOI: http://dx.doi.org/10.33448/rsd-v10i15.23077

Paula, A. A. D. (2020). Violência, descaso e/ou desconhecimento? A multiplicidade de relações entre o estado (de Mato Grosso do Sul) e os/as ostomizados. 84 f. Dissertação 84 f. Dissertação (Mestrado em Sociologia) - Universidade Federal da Grande Dourados.

Silva, J. A. G. (2020). Estomaterapia-a importância da atuação do profissional de enfermagem no suporte clínico e emocional de pacientes. Revista Feridas, (42), $1520-1520$.

Simon, B. S. (2020). Fortalecendo-se para seguir a vida: experiência de famílias ao conviver com familiar adulto com estomia por câncer intestinal. Doctoral dissertation, Universidade Federal de Santa Maria.

Vieira, S. A. M. (2018). Estomia de eliminação intestinal: dois lados de uma mesma história. Universidade Federal de Mato Grosso, Instituto de Ciências da Saúde, Sinop, 87.

Wojastyk, L. D. M. C., de Paula, M. A. B., \& Prado, M. N. B. (2020). Estomaterapia: influências e repercurssões na carreira profissional. Estima-Brazilian Journal of Enterostomal Therapy, 18. 